\title{
Transjugular Intrahepatic Portosystemic Shunt: Indications, Contraindications, and Patient Work-Up
}

\author{
Alexander Copelan, MD ${ }^{1}$ Baljendra Kapoor, MD, FSIR ${ }^{2}$ Mark Sands, MD, FACR ${ }^{2}$ \\ ${ }^{1}$ Diagnostic Radiology Department, William Beaumont Hospital, Royal \\ Oak, Michigan \\ 2 Imaging Institute, Cleveland Clinic, Cleveland, Ohio \\ Address for correspondence Alexander Copelan, MD, Diagnostic \\ Radiology Department, William Beaumont Hospital, 3601 W 13 Mile Road, \\ Royal Oak, MI 48073 (e-mail: alexander.copelan@beaumont.edu).
}

Semin Intervent Radiol 2014;31:235-242

\begin{abstract}
Keywords

- transjugular intrahepatic portosystemic shunt

- indications

- contraindications

- portal hypertension

- interventional radiology

The transjugular intrahepatic portosystemic shunt (TIPS) procedure is effective in achieving portal decompression and in managing some of the major complications of portal hypertension. While many clinicians are familiar with the two most common indications for TIPS placement, secondary prophylaxis of esophageal variceal hemorrhage and treatment of refractory ascites, evidence for its usefulness is growing in other entities, where it has been less extensively studied but demonstrates promising results. Newer indications include early utilization in the treatment of esophageal variceal hemorrhage, Budd-Chiari syndrome, ectopic varices, and portal vein thrombosis. The referring clinician and interventionist must remain cognizant of the contraindications to the procedure to avoid complications and potential harm to the patient. This review is designed to provide an in-depth analysis of the most common as well as less typical indications for TIPS placement, and to discuss the contraindications and appropriate patient evaluation for this procedure.
\end{abstract}

Objectives: Upon completion of this article, the reader will be able to describe the indications, contraindications, and patient work-up algorithm for placement of a TIPS.

Accreditation: This activity has been planned and implemented in accordance with the Essential Areas and Policies of the Accreditation Council for Continuing Medical Education (ACCME) through the joint providership of Tufts University School of Medicine (TUSM) and Thieme Medical Publishers, New York. TUSM is accredited by the ACCME to provide continuing medical education for physicians.

Credit: Tufts University School of Medicine designates this journal-based CME activity for a maximum of 1 AMA PRA Category 1 Credit $^{\mathrm{TM}}$. Physicians should claim only the credit commensurate with the extent of their participation in the activity.

Originally described by Rösch et al in 1969 as a "radiologic portocaval shunt," the transjugular intrahepatic portosyste- mic shunt (TIPS) procedure is a minimally invasive nonsurgical method of achieving portal decompression to treat some of the major complications of portal hypertension. ${ }^{1}$ Typically performed by an interventional radiologist under fluoroscopic guidance and general anesthesia, it is advantageous over the surgical portocaval shunt in that it does not subject the patient to a large abdominal incision and potentially decreases the morbidity and length of hospital stay.

The indications for TIPS with the most evidence-based support are secondary prophylaxis of esophageal variceal hemorrhage and treatment of refractory ascites. Indications are continuously expanding, however, and it is essential that not only the interventional radiologist but also the referring physician be knowledgeable regarding its appropriate use. This review focuses on the indications as well as the contraindications and appropriate patient evaluation for TIPS placement, to ensure that desired clinical results can be achieved while minimizing post-TIPS complications.
Issue Theme TIPS; Guest Editor, Wael E. Saad, MD, FSIR
Copyright @ 2014 by Thieme Medical Publishers, Inc., 333 Seventh Avenue, New York, NY 10001, USA. Tel: +1(212) 584-4662.
DOI http://dx.doi.org/ 10.1055/s-0034-1382790. ISSN 0739-9529. 
Table 1 Indications for TIPS related to variceal bleeding

\begin{tabular}{|l|l|}
\hline Indications & Role of TIPS \\
\hline $\begin{array}{l}\text { Secondary prophylaxis of } \\
\text { variceal bleeding }\end{array}$ & Rescue therapy $^{\mathrm{a}}$ \\
\hline Acute variceal bleeding & Rescue therapy $^{\mathrm{a}}$ \\
\hline Portal hypertensive gastropathy & Rescue therapy $^{\mathrm{a}}$ \\
\hline Recurrent acute variceal bleeding & First-line therapy $^{\mathrm{a}}$ \\
\hline
\end{tabular}

Abbreviations: HVPG, hepatic venous pressure gradient; TIPS, transjugular intrahepatic portosystemic shunt.

Note: Gastric varices might require variceal embolization.

${ }^{\mathrm{a} A p p l i e s}$ to esophageal and ectopic varices.

\section{Indications}

The two indications with the strongest clinical evidence of TIPS efficacy are secondary prevention of esophageal variceal bleeding and treatment of refractory ascites. Meta-analyses and multiple randomized controlled trials support TIPS in both circumstances. Additional indications, some of which are emerging as controversial first-line treatments, are listed in -Tables $\mathbf{1}$ and $\mathbf{2}$ and described in detail below. ${ }^{2}$ Firm indications are likely to expand in the future.

\section{Prevention of Variceal Rebleeding}

Strong evidence supports the use of TIPS for secondary prevention of esophageal variceal bleeding (-Fig. 1). This indication has been extensively studied, and TIPS has been compared with alternative forms of therapy in 13 randomized controlled trials (which describe results for 948 patients) and several meta-analyses. A critical guideline is that a post-TIPS portosystemic pressure gradient (PPG) less than $12 \mathrm{~mm} \mathrm{Hg}$ should be obtained to prevent a rebleeding episode. ${ }^{3}$

A meta-analysis performed in 1999 clearly demonstrated that TIPS is substantially more effective in long-term prevention of rebleeding than is endoscopic therapy (19\% incidence of rebleeding vs. $47 \%$, respectively). ${ }^{4} \mathrm{~A}$ more recent metaanalysis confirmed TIPS advantage in prevention of rebleeding in comparison to endoscopic therapy (19.0\% incidence of

Table 2 Indications for TIPS

\begin{tabular}{|l|}
\hline $\begin{array}{l}\text { Variceal bleeding, secondary prevention, and acute } \\
\text { bleeding refractory to medical and endoscopic treatments }\end{array}$ \\
\hline Refractory ascites \\
\hline Hepatorenal syndrome (types 1 and 2 ) \\
\hline Budd-Chiari syndrome \\
\hline Hepatic veno-occlusive disease \\
\hline Hepatic hydrothorax \\
\hline Portal hypertensive gastropathy \\
\hline Hepatopulmonary syndrome \\
\hline Portal vein thrombosis \\
\hline
\end{tabular}

Abbreviation: TIPS, transjugular intrahepatic portosystemic shunt.

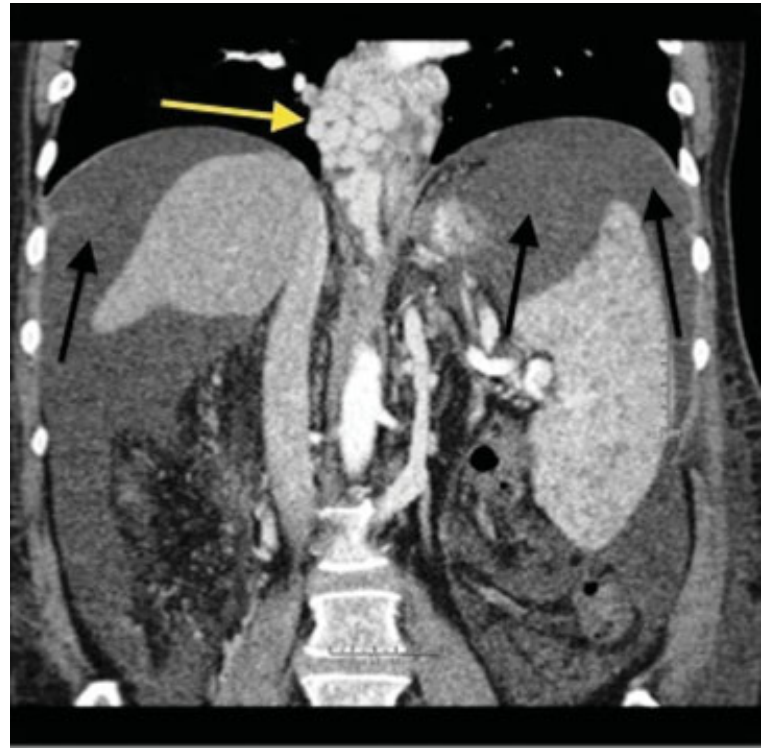

Figure 1 Coronal contrast-enhanced computed tomography of the abdomen demonstrates avidly enhancing, markedly tortuous, and dilated varices surrounding the lower esophagus (yellow arrow). There is also significant perihepatic and perisplenic ascites (black arrows). The spleen is enlarged secondary to portal hypertension.

rebleeding vs. $43.8 \%$, respectively). ${ }^{5}$ The reproducibility of data over the course of two decades is convincing. Further, a large retrospective study demonstrated that variceal embolization at the time of TIPS significantly prevented the rate of recurrent variceal bleeding in comparison to TIPS alone (84 vs. $61 \%$ at 2 years; 81 vs. $53 \%$ at 4 years, respectively). ${ }^{6}$ Allcause mortality rates are similar between TIPS and endoscopic therapy groups.

Unfortunately, TIPS is associated with a significantly higher rate of development of hepatic encephalopathy (HE) than standard therapy in the management of esophageal varices. ${ }^{7}$ Due to this drawback, many advocate that TIPS be used predominately as a backup to endoscopic therapy for secondary prophylaxis of variceal bleeding.

TIPS and distal splenorenal shunt were reported to be equally efficacious with no difference in HE in the prevention of rebleeding at a follow-up of 2 to 8 years for patients with Child-Pugh A or B cirrhosis. ${ }^{8}$ This study also demonstrated an exceptionally low rebleeding rate following TIPS ( $10.5 \%$ at 48 months), and encouraging survival rates at 2 and 5 years of 88 and $61 \%$, respectively. While patients undergoing TIPS required more postprocedure interventions to maintain patency, the use of bare stents almost certainly contributed to this. Importantly, cost analysis favored TIPS. ${ }^{7}$ The 2009 American Association for the Study of Liver Disease (AASLD) updated guidelines judged these two approaches equally effective. ${ }^{9}$ These studies further emphasize that survival rates following TIPS vary widely depending on the primary indication for the procedure and the underlying health of the patient. Selection of appropriate patients is therefore of utmost importance in determining outcome. 
Early utilization of TIPS demonstrated promising results in a study by García-Pagán et al, who reported results of a prospective randomized study of 63 patients with cirrhosis and acute variceal bleeding at high risk of failure with standard treatment (Child-Pugh class C, with a score $<14$, or Child-Pugh class B, but with active bleeding at diagnostic endoscopy). ${ }^{10}$ All were initially treated with vasoactive drugs and endoscopic therapy. They were randomized within 24 hours of admission either: to a control group that received continued administration of vasoactive drugs, followed by 3 to 5 days of treatment with propranolol or naldol plus endoscopic band ligation (EBL) with insertion of TIPS reserved as rescue therapy; or to early TIPS (within 72 hours of randomization). At 16 months median follow-up, rebleeding or failure to control bleeding (14 patients vs. 1 patient, $p=0.001$ ) and death (12 patients vs. 4 patients, $p=0.01$ ) occurred significantly more frequently in the control group. No significant difference in serious adverse events was observed between the two groups. ${ }^{10}$

In this same study, 1 year actuarial survival was $61 \%$ in the pharmacologic-EBL group and $86 \%$ in the early TIPS group $(p<0.001)$. Seven patients in the control group, four of whom subsequently died, underwent rescue TIPS, raising the question: would more of the patients receiving rescue TIPS have survived had they received early TIPS? This prospective randomized controlled trial clearly demonstrates that early TIPS significantly reduces the incidence of treatment failure and early mortality in cirrhotic patients hospitalized for acute variceal bleeding identified as at high risk of failure with standard treatment.

In a separate study, Monescillo et al demonstrated that patients with acute variceal bleeds and a PPG $>20 \mathrm{~mm} \mathrm{Hg}$ benefit from early TIPS intervention. ${ }^{11}$ TIPS should therefore be considered for first-line treatment of patients identified as likely to fail standard initial treatment.

\section{Refractory Ascites}

Six randomized controlled trials including a total of 390 patients, and two meta-analyses, have confirmed the efficacy of TIPS in the treatment of refractory ascites (-Fig. 1). TIPS provides a logical approach to reducing the formation of ascites by correcting two major pathophysiologic determinants of ascites: first, it lowers the elevated sinusoidal pressure that contributes significantly to the formation of ascites, and second, TIPS leads to increased effective arterial blood volume, which in turn leads to a downregulation of the renin-angiotensin-aldosterone axis and increased natriuresis. ${ }^{12}$ TIPS also leads to increased venous return and increased cardiac output, which increases the arterial pressure and the glomerular filtration rate; therefore, the functional renal failure that occurs with and further promotes refractory ascites may also be corrected.

A meta-analysis of four randomized controlled trials compared TIPS to large-volume paracentesis in the treatment of refractory ascites. ${ }^{13}$ TIPS was effective in controlling ascites in $70 \%$ of cases, more than three times the $23 \%$ rate observed with large-volume paracentesis. A meta-analysis in 2005 showed a 7.1-fold reduction in the risk of recurrence of tense ascites after TIPS. ${ }^{14}$

TIPS alone may not be sufficient for control of refractory ascites; many patients still require short-term diuretics following the procedure as natriuresis slowly but steadily increases over the course of up to 1 year. Compared with large-volume paracentesis, TIPS improved transplant-free survival and the incidence of recurrent ascites in cirrhotic patients with refractory ascites. ${ }^{13}$ Survival of patients in this report was independently associated with age, bilirubin levels, and serum sodium concentration. The probability of HE was significantly increased following TIPS. Patients with low arterial pressure, high Model for End-Stage Liver Disease (MELD) score, and a low PPG after TIPS had the greatest probability of experiencing post-TIPS encephalopathy, again emphasizing the importance of proper patient evaluation and selection in determining outcome. ${ }^{13}$

$\mathrm{HE}$ is the major adverse consequence of TIPS. Patients with refractory ascites typically have severe cirrhosis, and 40\% develop HE following TIPS. ${ }^{9}$ In a randomized controlled trial comparing TIPS to paracentesis plus albumin, in which the majority of patients were in the Child-Pugh $C$ group and therefore at greater risk of $\mathrm{HE}$, significantly better survival without the need for liver transplant was achieved in those undergoing TIPS. ${ }^{15}$ Because TIPS is associated with an increased risk of HE and higher cost, many experts consider repeat large-volume paracentesis to be the treatment of choice. However, large-volume paracentesis is not without its limitations. Paracentesis does not prevent recurrence of ascites and can instigate worsening circulatory dysfunction, leading to the dreaded complication of hepatorenal syndrome (HRS). Paracentesis also carries the risk of spontaneous bacterial peritonitis and incarceration of abdominal hernias.

The PPG required for effective control of refractory ascites in most patients is uncertain; a gradient $<8 \mathrm{~mm} \mathrm{Hg}$ has been suggested based on limited data. ${ }^{16}$ Initially aiming for higher gradients may limit worsening of encephalopathy in individuals with pre-existing HE, providing the potential for later enlargement of the TIPS if HE is adequately controlled and diuresis is inadequate. ${ }^{8}$

As with other indications for the TIPS procedure, patient selection is paramount. Early use of TIPS in selected candidates appears reasonable. ${ }^{16}$ Regarding patient selection, the pre-TIPS bilirubin level is the parameter most clearly associated with increased mortality. ${ }^{16}$

\section{Acute Variceal Bleeding}

TIPS is established as second-line therapy for acute variceal bleeding when medical management, including pharmacologic and endoscopic therapy, has failed. The combination of vasoactive drugs and endoscopic sclerotherapy is generally quite effective, achieving hemostasis in $>80 \%$ of cases. ${ }^{17}$ TIPS is more effective, controlling acute bleeding in 95\% of cases, with a rebleeding rate of just $18 \%$, but due to post-TIPS encephalopathy, the need for post-TIPS evaluations, and the cost of the procedure, many experts consider the TIPS procedure appropriate only as rescue therapy. ${ }^{9}$ 
Results of surgical portocaval shunting must also be considered. Operative shunts, such as distal splenorenal shunt, may be preferred in some patients in the Child-Pugh A group because fewer postoperative interventions are required to ensure patency than seen with TIPS. However, over the last two decades, the use of PTFE-covered stent grafts in patients with TIPS has significantly reduced reintervention rates, resulting in reduction in cost burden on the health care system. ${ }^{7}$ Child-Pugh C patients are generally unable to tolerate general anesthesia or surgery, and since TIPS may be performed without general anesthesia, TIPS provides these patients with a unique opportunity for definitive treatment.

\section{Acute Ectopic Variceal Bleeding}

Ectopic varices comprise 1 to $5 \%$ of variceal bleeds in patients with intrahepatic portal hypertension secondary to cirrhosis, and 20 to $30 \%$ of those with extrahepatic portal hypertension (-Fig. 2). ${ }^{18}$

Since TIPS directly decompresses portal pressure, which is the cause of variceal rupture (whether esophageal, gastric, intestinal, peritoneal, or stomal), patients with all forms of varices should benefit from the portal pressure decompression achieved with TIPS. Patients with varices inaccessible by means of endoscopy in whom banding or injection cannot be performed particularly benefit from portal decompression procedures.

Gastric variceal bleeds require particular attention as they have a worse outcome than esophageal variceal bleeds, with a mortality rate of 45 to $55 \%$ ( - Fig. 3 ). ${ }^{19}$ Since gastric varices rebleed at even lower portal pressures following TIPS, additional therapy, such as variceal embolization at the time of the TIPS, may be necessary. ${ }^{9}$ Variceal embolization has also been achieved using the balloon-occluded retrograde obliteration technique via gastrorenal shunts. Periodic surveillance of these patients is vital since small increases in portal pressure may cause rebleeding. ${ }^{20}$

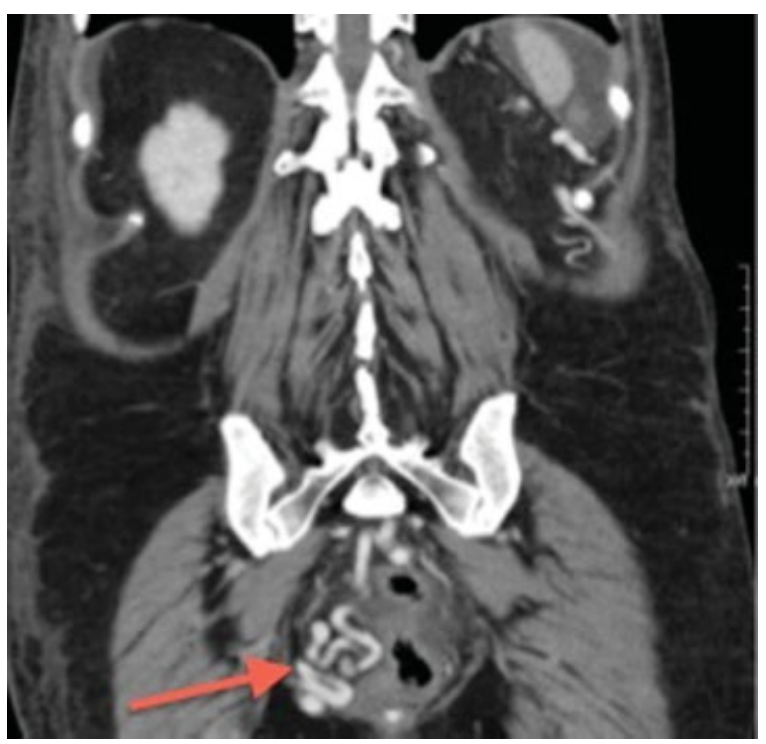

Figure 2 Coronal contrast-enhanced computed tomography demonstrates avidly enhancing tortuous and dilated rectal (hemorrhoidal) varices (arrow).

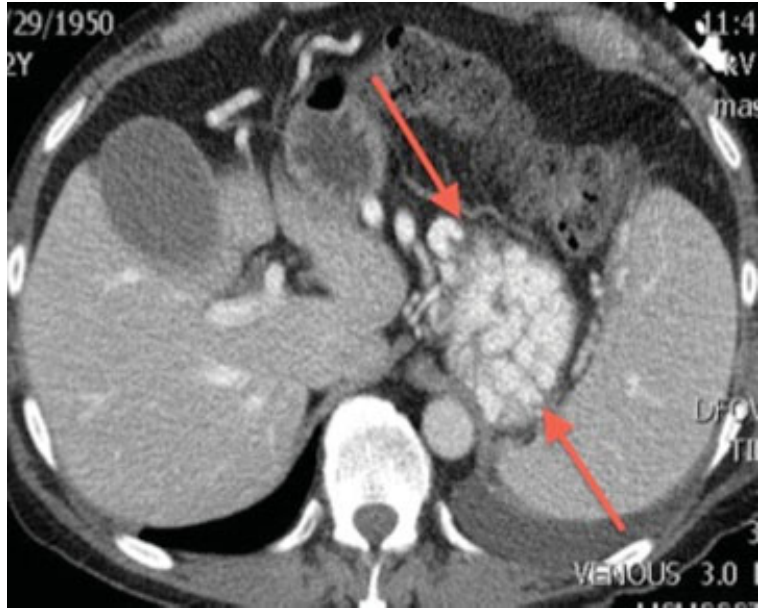

Figure 3 Axial contrast-enhanced computed tomography image through the abdomen demonstrates enhancing, tortuous dilated varices (arrows) in the region of the gastric fundus.

\section{Budd-Chiari Syndrome}

TIPS is often more technically challenging in Budd-Chiari syndrome (BCS) as there may be absence of normal hepatic veins to use as a starting point for the initial puncture. A small segment of the origin of a hepatic vein from the inferior vena cava (IVC), or a "stump," is sometimes discovered at IVC venography. If the stump can be accessed, the liver can be punctured via the stump and carbon dioxide portal venography can assist in locating the portal venous system for subsequent access. In the case of inaccessible hepatic veins secondary to occlusion of the ostia, a direct shunt between the retrohepatic vena cava and portal vein may be established.

In patients presenting weeks to months after the initial formation of hepatic vein thrombosis, the thrombus may no longer be amenable to anticoagulation alone, nor to interventional procedures such as angioplasty or thrombolysis. TIPS is recommended as the next step in management. ${ }^{21}$ In those who have already failed thrombolytic therapy, and in those with poor hepatic reserve, an occluded IVC, or a portal vein-infrahepatic vena caval pressure gradient more than $10 \mathrm{~mm} \mathrm{Hg}$, TIPS is also recommended.

At European centers, TIPS is the most common form of intervention for BCS. Multiple studies have demonstrated its technical success as well as its relatively low rate of complications. ${ }^{22}$ Rössle et al demonstrated an initial technical success in 33 out of 35 patients, followed by 1and 5-year transplant-free survival rates of 93 and $74 \%$, respectively. ${ }^{23}$

In another series of patients with BCS who failed to improve with anticoagulation, patients who had TIPS had transplant-free survival rates at 1 year of $88 \%$ and at 10 years of $69 \%$, which were better than predicted. ${ }^{24}$ TIPS patency was superior in those receiving covered stents.

The success of TIPS in the emergent acute setting of BCS, or "rescue TIPS," has also been validated. ${ }^{25}$ TIPS may also serve as a bridge to liver transplant in this patient population. ${ }^{22}$ 
TIPS has the advantage over surgical side-to-side portocaval, mesocaval, and splenorenal portosystemic shunts in that it may bypass caval stenosis or thrombus. Like a mesoatrial shunt, TIPS provides outflow into the suprahepatic IVC, at a site proximal to a potential intrahepatic stenosis. As patients with BCS are typically hypercoagulable, simultaneous portal vein thrombosis (PVT) may be present; extended TIPS have been described in such cases and have demonstrated success. ${ }^{26}$

The 2009 guidelines recommended TIPS in those patients with BCS who fail to improve with anticoagulation alone. ${ }^{8}$

\section{Portal Vein Thrombosis}

PVT is characterized by the presence of a completely or partially obstructing thrombus within the main portal vein or its intrahepatic portal branches, and can involve the portal tributaries including the splenic vein or superior mesenteric vein (SMV). PVT occurs in 4.4 to $15 \%$ of cirrhotic patients. ${ }^{27}$ Hypercoagulable states, tumors, infection, pancreatitis, and trauma are common underlying causes of noncirrhotic PVT. Early detection of PVT permits timely intervention and prevention of complications, including variceal hemorrhage and intestinal ischemia. The creation of a TIPS can be technically challenging in the presence of PVT, especially when the thrombus is chronic and cavernous transformation of the portal vein has occurred.

Several approaches, including transhepatic, transjugular, transmesenteric using a combined surgical approach, and transsplenic for recanalization of the portal vein, have been described. ${ }^{28}$ Advanced imaging tools, such as cone beam computed tomography and iGuide (Syngo iGuide; Siemens Healthcare, Forchheim, Germany), are very useful in accessing patent segments of the portal venous system in patients with PVT and cavernous transformation of the portal vein. Wedged hepatic venography using carbon dioxide may better define portal venous anatomy in cases of PVT. Once a puncture is performed, a guidewire is used as a probe in an attempt to access the main portal vein. Portography obtained via an angiographic catheter can confirm position. Interventions including pulse-spray thrombolysis, reverse Fogarty maneuver, mechanical thrombectomy, or stent placement can be implemented. Once the portal clot is maximally removed, the TIPS can be created.

Stating that "There is no established management algorithm for PVT in cirrhotic patients," Senzolo et al prospectively compared a control group of patients with nonmalignant PVT who neither were anticoagulated nor had received TIPS, versus a study group, in whom administration of low-molecular-weight heparin (LMWH) was planned. $^{29}$ If the patient had a contraindication to anticoagulation or the PVT progressed while on LMWH, the patient was designated to undergo TIPS. The use of anticoagulation and TIPS improved the chance of complete reperfusion, reduced portal hypertensive complications (mainly variceal bleeding and intestinal venous ischemic episodes), and decreased the rate of thrombus progression. Blum et al demonstrated that TIPS and recanalization of the main portal vein is a safe and effective treatment for patients with cirrhosis and noncavernous portal vein occlusion. ${ }^{30}$ The presence of portal cavernous transformation and the degree of thrombus within the main portal vein, the portal vein branches, and the SMV are independent predictors of success. ${ }^{31}$

In PVT in noncirrhotic patients, studies have clearly demonstrated that anticoagulation is safe and effective and the therapy of choice. ${ }^{32}$ Anticoagulation prevents not only rethrombosis but also extension of thrombus into the portal venous system, thereby preventing an increase in portal pressure. In approximately $10 \%$ of cases of acute PVT in noncirrhotics, however, the thrombus is resistant to anticoagulation. ${ }^{33}$ For chronic PVT in noncirrhotics, anticoagulant treatment is administered to only $30 \%$ of patients, reflecting concerns about the use of anticoagulation in the presence of gastroesophageal varices, low platelet counts, and impaired coagulation. ${ }^{34}$ Two separate studies demonstrated the effectiveness of combined TIPS and localized thrombolytic therapy in noncirrhotic patients with symptomatic massive PVT. ${ }^{35,36}$

\section{Hepatic Veno-occlusive Disease}

Following hematopoietic cell transplantation, veno-occlusive disease of the liver is a life-threatening complication. Liver failure and acute portal hypertension are frequently present. In a case series of 10 patients with veno-occlusive disease, TIPS controlled portal hypertension in all 10 patients, supporting consideration of early TIPS to improve survival rates in those patients with veno-occlusive disease. ${ }^{37}$

\section{Hepatic Hydrothorax}

Several retrospective case series support the use of TIPS in the setting of hepatic hydrothorax, defined as a significant pleural effusion, typically larger than $500 \mathrm{~mL}$, in a cirrhotic patient without primary pulmonary or cardiac disease. ${ }^{38}$ Approximately $5 \%$ of cirrhotic patients are affected with hepatic hydrothorax. ${ }^{38}$ Complete resolution of hydrothorax occurs in 57 to $71 \%$ of patients, and at least partial improvement in dyspnea and decrease in frequency of thoracenteses has been reported in 68 to $82 \%$ of patients. ${ }^{38,39}$

\section{Hepatorenal Syndrome}

TIPS significantly reduces the PPG, significantly improves renal function within 2 weeks of the procedure (creatinine clearance from 18 to $48 \mathrm{~mL} / \mathrm{min}$ ), and improves survival rates of both types I and II HRS. ${ }^{40}$ Serum bilirubin level and HRS subtype are independent predictors of survival following TIPS. ${ }^{40}$

\section{Hepatopulmonary Syndrome}

In a small series, TIPS neither improved nor worsened pulmonary gas exchange in patients with hepatopulmonary syndrome (HPS). ${ }^{41}$ While this does not directly support TIPS in HPS, it demonstrates its relative safety when performed for the treatment of other complications of portal hypertension in patients with HPS.

\section{Portal Hypertensive Gastropathy}

Portal hypertensive gastropathy (PHG) is characterized by an endoscopic abnormality of the gastric mucosa that is classically described as a "snakeskin" pattern, with or without red 
spots, typically located in the fundus or body of the stomach. $^{42}$

In patients with PHG, level 4 evidence in the form of case series supports the usefulness of TIPS. ${ }^{43}$ TIPS improves endoscopic findings of PHG, and in one patient with massive hematemesis hemorrhage completely stopped following TIPS. The mechanism by which PHG improves following TIPS may be closely related to the improvement of the injured gastric perfusion in cirrhotic patients with PHG.

\section{Contraindications}

The contraindications to TIPS are best appreciated with an understanding of its hemodynamic consequences.

\section{Hemodynamic Consequences of Portosystemic Shunting}

Creation of a TIPS shunts ammonia and other neurotoxins directly into the systemic circulation, increasing the incidence of HE. HE often occurs soon after TIPS (90\% within the first 3 months), before the apparent eventual cerebral adaptation to gut-derived neurotoxins. A major risk factor for postprocedural development of $\mathrm{HE}$ is a prior history of $\mathrm{HE}^{44}$

As a consequence of TIPS, hepatic arterial flow assumes increased responsibility for sinusoidal perfusion since portal flow normally supplies $60 \%$ of oxygen delivery to the liver. In some patients, such as those with hepatic artery stenosis, hepatic artery flow may be insufficient to adequately compensate for the lack of portal flow, resulting in relative ischemia and progressive hepatic failure. ${ }^{45}$

In decompressing the portal system, portal blood is shunted to the systemic circulation, leading to increased systemic venous blood return. The post-TIPS elevation in preload can precipitate heart failure not only in patients with pre-existing overt heart failure or severe tricuspid regurgitation but also in patients with an underlying subclinical cardiac insufficiency. The increased preload may also worsen undiagnosed portopulmonary hypertension.

\section{Absolute and Relative Contraindications}

Several pre-existing clinical conditions increase the likelihood of an unfavorable outcome following TIPS ( - Table 3 ). Absolute contraindications to TIPS placement include severe pulmonary hypertension (mean pulmonary pressure $>45 \mathrm{~mm} \mathrm{Hg}$ ), severe tricuspid regurgitation, congestive heart failure, severe liver failure, and polycystic liver disease. Also, no patients with active sepsis should undergo TIPS. Relative contraindications include severe obstructive arteriopathy, and hepatic artery and celiac trunk stenosis, which may prevent adequate sinusoidal perfusion by the hepatic artery. Other relative contraindications include recurrent $\mathrm{HE}$, hepatocellular carcinoma and other liver tumors, and bile duct dilation.

\section{Patient Work-Up}

Determining whether or not a patient should undergo a TIPS procedure should be a team-based decision involving the hepatologist or gastroenterologist caring for the patient as
Table 3 Contraindications to placement of a TIPS

\begin{tabular}{|l|l|}
\hline Absolute & Relative \\
\hline $\begin{array}{l}\text { Primary prevention } \\
\text { of variceal bleeding }\end{array}$ & $\begin{array}{l}\text { Hepatoma, particularly } \\
\text { if central }\end{array}$ \\
\hline $\begin{array}{l}\text { Severe congestive } \\
\text { heart failure }\end{array}$ & $\begin{array}{l}\text { Obstruction of all } \\
\text { hepatic veins }\end{array}$ \\
\hline Tricuspid regurgitation & Hepatic encephalopathy \\
\hline Multiple hepatic cysts & $\begin{array}{l}\text { Significant portal vein } \\
\text { thrombosis }\end{array}$ \\
\hline $\begin{array}{l}\text { Uncontrolled systemic } \\
\text { infection or sepsis }\end{array}$ & $\begin{array}{l}\text { Severe uncorrectable } \\
\text { coagulopathy (INR }>5)\end{array}$ \\
\hline $\begin{array}{l}\text { Unrelieved biliary } \\
\text { obstruction }\end{array}$ & $\begin{array}{l}\text { Thrombocytopenia } \\
\text { (<20,000 platelets } / \mathrm{mm}^{3} \text { ) }\end{array}$ \\
\hline $\begin{array}{l}\text { Severe pulmonary } \\
\text { hypertension }\end{array}$ & $\begin{array}{l}\text { Moderate pulmonary } \\
\text { hypertension }\end{array}$ \\
\hline
\end{tabular}

Abbreviations: INR, international normalized ratio; TIPS, transjugular intrahepatic portosystemic shunt.

well as the interventional radiologist. A general anesthesia consultation is typically required. Several clinical questions must first be answered, including: Is TIPS indicated for treatment of this specific complication of portal hypertension? Are absolute or relative contraindications present? Does the patient have a history of HE? What is the patient's MELD score?

Post-TIPS HE occurs more commonly in cirrhotic patients with refractory ascites than in those undergoing TIPS for variceal bleeding, suggesting that patients with severe cirrhosis are more likely to experience encephalopathy. This factor should be considered in the selection of patients for the TIPS procedure. ${ }^{12}$ The main risk factors for developing HE include age $>65$ years, Child score $>12$, prior HE, placement of a large diameter stent $(>10 \mathrm{~mm})$, and low PPG $(<5 \mathrm{~mm}$ $\mathrm{Hg}) .{ }^{44}$ The risk of HE may outweigh the potential benefit of the procedure in patients possessing these risk factors.

One series reported that careful selection of patients who received TIPS for variceal bleeding, and aiming for a PPG reduction to just below the $12 \mathrm{~mm} \mathrm{Hg}$ threshold required to prevent rebleeding, led to post-TIPS HE that was usually short-lived and easily managed (i.e., withdrawal of diuretics or psychotropic medication, commencement of lactulose, use of antimicrobials). ${ }^{44}$ While this study reported an HE incidence of $34.5 \%$, the majority of the cases were "low grade" and resolved with conservative management. Since HE can also be precipitated by variceal bleeding, arrest of bleeding with TIPS can actually improve encephalopathy.

The MELD score is calculated based on the patients creatinine, bilirubin, and international normalized ratio (INR), and has proven superior to the Child-Pugh score at predicting post-TIPS mortality. ${ }^{46}$ A MELD score above 18 predicts a significantly higher mortality 3 months after TIPS in comparison to those with a MELD score of 18 or lower. ${ }^{46}$

If the patient is deemed an appropriate candidate for TIPS, a comprehensive clinical history and physical exam are necessary. Within 24 hours of the TIPS procedure, a complete 
blood count, comprehensive metabolic panel, liver function tests, and coagulation profile should be obtained. Appropriate blood products should be administered if there is significant coagulopathy (INR $>1.5$ ), thrombocytopenia (platelets $<50,000 / \mathrm{mm}^{3}$ ), or anemia (hematocrit $<25 \%$ ).

An echocardiogram should be obtained in patients with known pulmonary or cardiac disease. If recent cross-sectional imaging is available, such as contrast-enhanced cross-sectional imaging or Doppler ultrasound, this should be reviewed. If no hepatic imaging within the past month is available, or if the patient has experienced recent deterioration in liver function, portal vein patency should be evaluated with a Doppler ultrasound.

Large-volume paracentesis or thoracentesis may be performed the day before or the day of the TIPS procedure for patients with refractory ascites and hepatic hydrothorax, respectively.

\section{Conclusion}

While TIPS is one of the more complex procedures performed by interventional radiologists, in appropriate clinical situations its benefits far exceed its associated risks. TIPS has gained increasing popularity due to its capacity to treat the major complications of portal hypertension in a nonsurgical manner and is effective in portal decompression in over 95\% of cases. TIPS can be successful in patients with severe cirrhosis who were untreatable 20 to 30 years ago.

The indications with the strongest clinical evidence of efficacy are secondary prevention of esophageal variceal bleeding and treatment of refractory ascites. Recently, a prospective randomized study has provided evidence that early TIPS should be considered for acute esophageal variceal bleeding in selected patients who are likely to fail standard treatment. ${ }^{10}$ TIPS is also currently used for other indications, including BCS, nonesophageal variceal bleeding, and hepatic hydrothorax, where evidence of its effectiveness and its appropriate role is accumulating.

The role of TIPS in the management of portal hypertension is constantly evolving, and interventionists continue to gain more experience with its technicalities. Results are steadily improving as a result of improved criteria for patient selection, identification of contraindications to the procedure, technical improvements including covered stents, and greater experience in creating TIPS and monitoring patients following the procedure. TIPS may soon be considered first-line therapy in selected patients in whom it is currently considered only for rescue therapy, and new indications for its use will continue to be discovered.

\section{References}

1 Rösch J, Hanafee WN, Snow H. Transjugular portal venography and radiologic portacaval shunt: an experimental study. Radiology 1969;92(5):1112-1114

2 Colombato L. The role of transjugular intrahepatic portosystemic shunt (TIPS) in the management of portal hypertension. J Clin Gastroenterol 2007;41(Suppl 3):S344-S351
3 Garcia-Tsao G, Groszmann RJ, Fisher RL, Conn HO, Atterbury CE, Glickman M. Portal pressure, presence of gastroesophageal varices and variceal bleeding. Hepatology 1985;5(3):419-424

4 Papatheodoridis GV, Goulis J, Leandro G, Patch D, Burroughs AK. Transjugular intrahepatic portosystemic shunt compared with endoscopic treatment for prevention of variceal rebleeding: a meta-analysis. Hepatology 1999;30(3):612-622

5 Zheng M, Chen Y, Bai J, et al. Transjugular intrahepatic portosystemic shunt versus endoscopic therapy in the secondary prophylaxis of variceal rebleeding in cirrhotic patients: meta-analysis update. J Clin Gastroenterol 2008;42(5):507-516

6 Tesdal IK, Filser T, Weiss C, Holm E, Dueber C, Jaschke W. Transjugular intrahepatic portosystemic shunts: adjunctive embolotherapy of gastroesophageal collateral vessels in the prevention of variceal rebleeding. Radiology 2005;236(1):360-367

7 Boyer TD, Henderson JM, Heerey AM, et al; DIVERT Study Group. Cost of preventing variceal rebleeding with transjugular intrahepatic portal systemic shunt and distal splenorenal shunt. J Hepatol 2008;48(3):407-414

8 Henderson JM, Boyer TD, Kutner MH, et al; DIVERT Study Group. Distal splenorenal shunt versus transjugular intrahepatic portal systematic shunt for variceal bleeding: a randomized trial. Gastroenterology 2006;130(6):1643-1651

9 Boyer TD, Haskal ZJ. AASLD Practice Guideline. The role of transjugular intra-hepatic portosystemic shunt in the management of portal hypertension. Hepatology 2005;41:1-15

10 García-Pagán JC, Caca K, Bureau C, et al; Early TIPS (Transjugular Intrahepatic Portosystemic Shunt) Cooperative Study Group. Early use of TIPS in patients with cirrhosis and variceal bleeding. N Engl J Med 2010;362(25):2370-2379

11 Monescillo A, Martínez-Lagares F, Ruiz-del-Arbol L, et al. Influence of portal hypertension and its early decompression by TIPS placement on the outcome of variceal bleeding. Hepatology 2004;40(4):793-801

12 Garcia-Tsao G. The transjugular intrahepatic portosystemic shunt for the management of cirrhotic refractory ascites. Nat Clin Pract Gastroenterol Hepatol 2006;3(7):380-389

13 Salerno F, Cammà C, Enea M, Rössle M, Wong F. Transjugular intrahepatic portosystemic shunt for refractory ascites: a metaanalysis of individual patient data. Gastroenterology 2007;133(3): 825-834

14 D’Amico G, Luca A, Morabito A, Miraglia R, D’Amico M. Uncovered transjugular intrahepatic portosystemic shunt for refractory ascites: a meta-analysis. Gastroenterology 2005;129(4):1282-1293

15 Salerno F, Merli M, Riggio O, et al. Randomized controlled study of TIPS versus paracentesis plus albumin in cirrhosis with severe ascites. Hepatology 2004;40(3):629-635

16 Rössle M, Gerbes AL. TIPS for the treatment of refractory ascites, hepatorenal syndrome and hepatic hydrothorax: a critical update. Gut 2010;59(7):988-1000

17 Garcia-Tsao G. Current management of the complications of cirrhosis and portal hypertension: variceal hemorrhage, ascites, and spontaneous bacterial peritonitis. Gastroenterology 2001; 120(3):726-748

18 Norton ID, Andrews JC, Kamath PS. Management of ectopic varices. Hepatology 1998;28(4):1154-1158

19 Sarin SK, Lahoti D, Saxena SP, Murthy NS, Makwana UK. Prevalence, classification and natural history of gastric varices: a longterm follow-up study in 568 portal hypertension patients. Hepatology 1992;16(6):1343-1349

20 Casado M, Bosch J, García-Pagán JC, et al. Clinical events after transjugular intrahepatic portosystemic shunt: correlation with hemodynamic findings. Gastroenterology 1998;114(6): 1296-1303

21 Plessier A, Sibert A, Consigny Y, et al. Aiming at minimal invasiveness as a therapeutic strategy for Budd-Chiari syndrome. Hepatology 2006;44(5):1308-1316 
22 Ryu RK, Durham JD, Krysl J, et al. Role of TIPS as a bridge to hepatic transplantation in Budd-Chiari syndrome. J Vasc Interv Radiol 1999;10(6):799-805

23 Rössle M, Olschewski M, Siegerstetter V, Berger E, Kurz K, Grandt D. The Budd-Chiari syndrome: outcome after treatment with the transjugular intrahepatic portosystemic shunt. Surgery 2004; 135(4):394-403

24 Garcia-Pagán JC, Heydtmann M, Raffa S, et al; Budd-Chiari Syndrome-Transjugular Intrahepatic Portosystemic Shunt Group. TIPS for Budd-Chiari syndrome: long-term results and prognostics factors in 124 patients. Gastroenterology 2008;135(3):808-815

25 Abujudeh H, Contractor D, Delatorre A, Koneru B. Rescue TIPS in acute Budd-Chiari syndrome. AJR Am J Roentgenol 2005;185(1):89-91

26 Pfammatter T, Benoit C, Cathomas G, Blum U. Budd-Chiari syndrome with spleno-mesenteric-portal thrombosis: treatment with extended TIPS. J Vasc Interv Radiol 2000;11(6):781-784

27 Amitrano L, Guardascione MA, Brancaccio V, et al. Risk factors and clinical presentation of portal vein thrombosis in patients with liver cirrhosis. J Hepatol 2004;40(5):736-741

28 Matsui O, Yoshikawa J, Kadoya M, et al. Transjugular intrahepatic portosystemic shunt after previous recanalization of a chronically thrombosed portal vein via a transmesenteric approach. Cardiovasc Intervent Radiol 1996;19(5):352-355

29 Senzolo M, M Sartori T, Rossetto V, et al. Prospective evaluation of anticoagulation and transjugular intrahepatic portosystemic shunt for the management of portal vein thrombosis in cirrhosis. Liver Int 2012;32(6):919-927

30 Blum U, Haag K, Rössle M, et al. Noncavernomatous portal vein thrombosis in hepatic cirrhosis: treatment with transjugular intrahepatic portosystemic shunt and local thrombolysis. Radiology 1995;195(1):153-157

31 Han G, Qi X, He C, et al. Tranjugular intrahepatic portosystemic shunt for portal vein thrombosis with symptomatic portal hypertension in liver cirrhosis. Dig Dis Sci 2012;57:1072-1082

32 Amitrano L, Guardascione MA, Scaglione M, et al. Prognostic factors in noncirrhotic patients with splanchnic vein thromboses. Am J Gastroenterol 2007;102(11):2464-2470

33 Plessier A, Murad SD, Hernandez-Guerra M. A prospective multicentric follow-up study on 105 patients with acute portal vein thrombosis (PVT): Results from the European network for vascular disorders of the liver (en-vie). Hepatology 2007;46:310A

34 Webster GJ, Burroughs AK, Riordan SM. Review article: portal vein thrombosis - new insights into aetiology and management. Aliment Pharmacol Ther 2005;21(1):1-9
35 Bilbao JI, Elorz M, Vivas I, Martínez-Cuesta A, Bastarrika G, Benito A. Transjugular intrahepatic portosystemic shunt (TIPS) in the treatment of venous symptomatic chronic portal thrombosis in non-cirrhotic patients. Cardiovasc Intervent Radiol 2004;27(5): 474-480

36 Kocher G, Himmelmann A. Portal vein thrombosis (PVT): a study of 20 non-cirrhotic cases. Swiss Med Wkly 2005;135(25-26): 372-376

37 Azoulay D, Castaing D, Lemoine A, Hargreaves GM, Bismuth H. Transjugular intrahepatic portosystemic shunt (TIPS) for severe veno-occlusive disease of the liver following bone marrow transplantation. Bone Marrow Transplant 2000;25(9):987-992

38 Dhanasekaran R, West JK, Gonzales PC, et al. Transjugular intrahepatic portosystemic shunt for symptomatic refractory hepatic hydrothorax in patients with cirrhosis. Am J Gastroenterol 2010; 105(3):635-641

39 Spencer EB, Cohen DT, Darcy MD. Safety and efficacy of transjugular intrahepatic portosystemic shunt creation for the treatment of hepatic hydrothorax. J Vasc Interv Radiol 2002;13(4):385-390

40 Brensing KA, Textor J, Perz J, et al. Long term outcome after transjugular intrahepatic portosystemic stent-shunt in non-transplant cirrhotics with hepatorenal syndrome: a phase II study. Gut 2000;47(2):288-295

41 Martinez-Palli G, Drake BB, Garcia-Pagan JC, et al. Effect of transjugular intrahepatic portosystemic shunt on pulmonary gas exchange in patients with portal hypertension and hepatopulmonary syndrome. World J Gastroenterol 2005;11(43):6858-6862

42 Thuluvath PJ, Yoo HY. Portal hypertensive gastropathy. Am J Gastroenterol 2002;97(12):2973-2978

43 Mezawa S, Homma H, Ohta $\mathrm{H}$, et al. Effect of transjugular intrahepatic portosystemic shunt formation on portal hypertensive gastropathy and gastric circulation. Am J Gastroenterol 2001; 96(4):1155-1159

44 Masson S, Mardini HA, Rose JD, Record CO. Hepatic encephalopathy after transjugular intrahepatic portosystemic shunt insertion: a decade of experience. QJM 2008;101(6):493-501

45 Hassoun Z, Deschênes M, Lafortune M, et al. Relationship between pre-TIPS liver perfusion by the portal vein and the incidence of post-TIPS chronic hepatic encephalopathy. Am J Gastroenterol 2001;96(4):1205-1209

46 Salerno F, Merli M, Cazzaniga M, et al. MELD score is better than Child-Pugh score in predicting 3-month survival of patients undergoing transjugular intrahepatic portosystemic shunt. J Hepatol 2002;36(4):494-500 